\title{
DESTRUCTION OF DIRTY ICE MANTLES BY SPUTTERING
}

\author{
P. A. AANNESTAD
}

Institute for Space Studies, Goddard Space Flight Center, NASA, N.Y., U.S.A.

\begin{abstract}
Dirty ice mantles are destroyed efficiently by sputtering of He atoms when clouds encounter shock velocities greater than $13-15 \mathrm{~km} \mathrm{~s}^{-1}$. Destruction due to grain-grain collisions is found to be about $10^{-3}$ times less efficient. Sputtering in the hot intercloud medium should make intercloud grains smaller than cloud grains.
\end{abstract}

We have studied the processes of grain destruction and molecule formation due to the sputtering of dirty ice grain mantles as the grains move relative to the gas in hot shock waves. Such waves are set up when interstellar clouds encounter expanding H II regions or when they collide with each other. The model for the grains is a core of either graphite or silicate with a radius of $0.05 \mu$ surrounded by a mantle composed primarily of $\mathrm{H}_{2} \mathrm{O}$ and $\mathrm{CH}_{4}$. The sputtering rates of these mantle materials have been calculated as a function of gas temperature and dust to gas velocity, employing a theory of oblique angle incidence sputtering. Helium is found to be the main sputtering agent, and the decoupling of gas and dust through the shock front is found to increase the sputtering yields substantially.

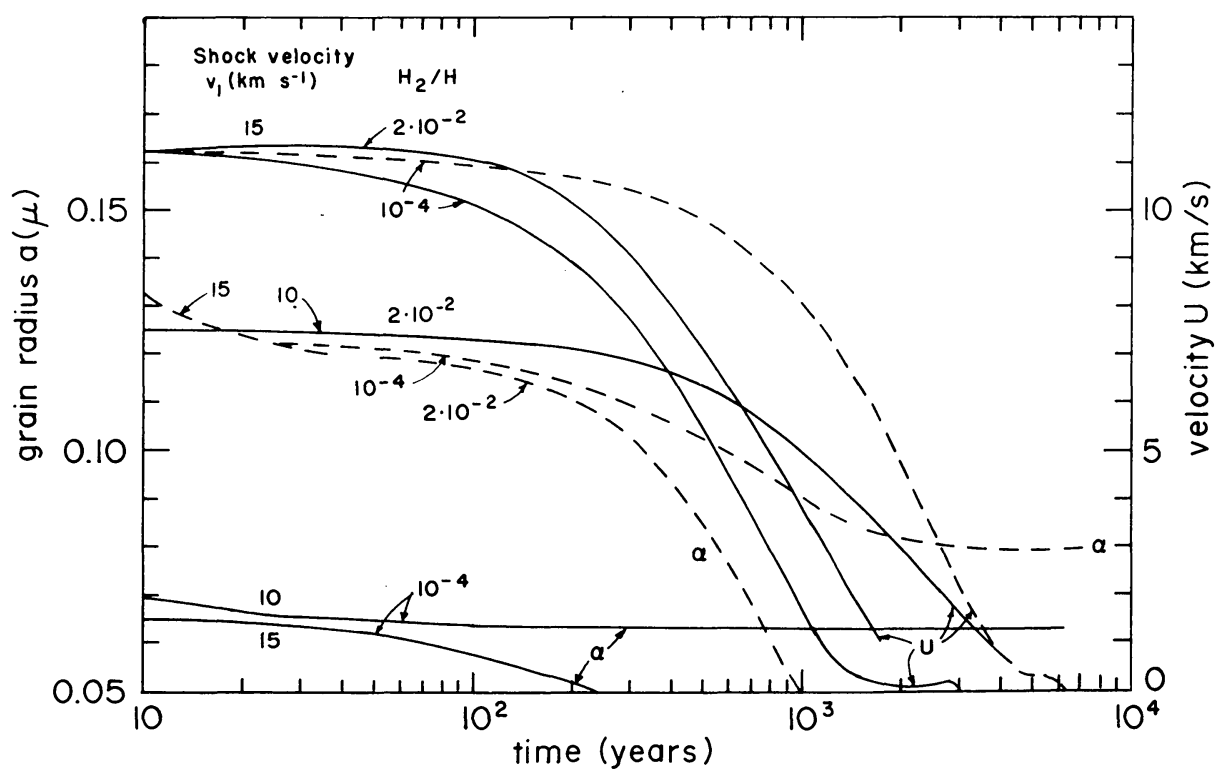

Fig. 1. The relative velocity between grain and gas and the grain radius as functions of time. The solid curves are for a cloud where the initial grain radius is $0.07 \mu$, and the dashed curves for a grain radius of $0.14 \mu$. 
In Figure 1 we show how the grain radius and the grain to gas velocity change as functions of time behind the shock front. The solid curves are for a cloud where the initial grain radius is about $0.07 \mu$, and the dashed curves for a grain radius of about $0.14 \mu$. We find that dirty ice mantles are destroyed efficiently by shock velocities greater than about $13-15 \mathrm{~km} \mathrm{~s}^{-1}$ if the abundance of molecular hydrogen is about one per cent or more, the corresponding life time of a mantle is about $5 \times 10^{7} \mathrm{yr}$. This leads to an equilibrium size distribution with an average grain size of $0.09 \mu-0.07 \mu$, the smallest value roughly satisfying the limit on the amount of ice in interstellar grains as found by Knacke et al. (1969).

In Figure 2 we show how the grain velocity, the relative grain density, the grain charge and the hydrogen density vary with the distance behind the shock front. The shock velocity is $10 \mathrm{~km} \mathrm{~s}^{-1}$ and $\mathrm{H}_{2} / \mathrm{H}$ is $2 \times 10^{-2}$. We see that the grains, starting out with a velocity of $10 \mathrm{~km} \mathrm{~s}^{-1}$ are decelerated to about $1 \mathrm{~km} \mathrm{~s}^{-1}$ within about $10^{-3} \mathrm{pc}$ from the shock front.

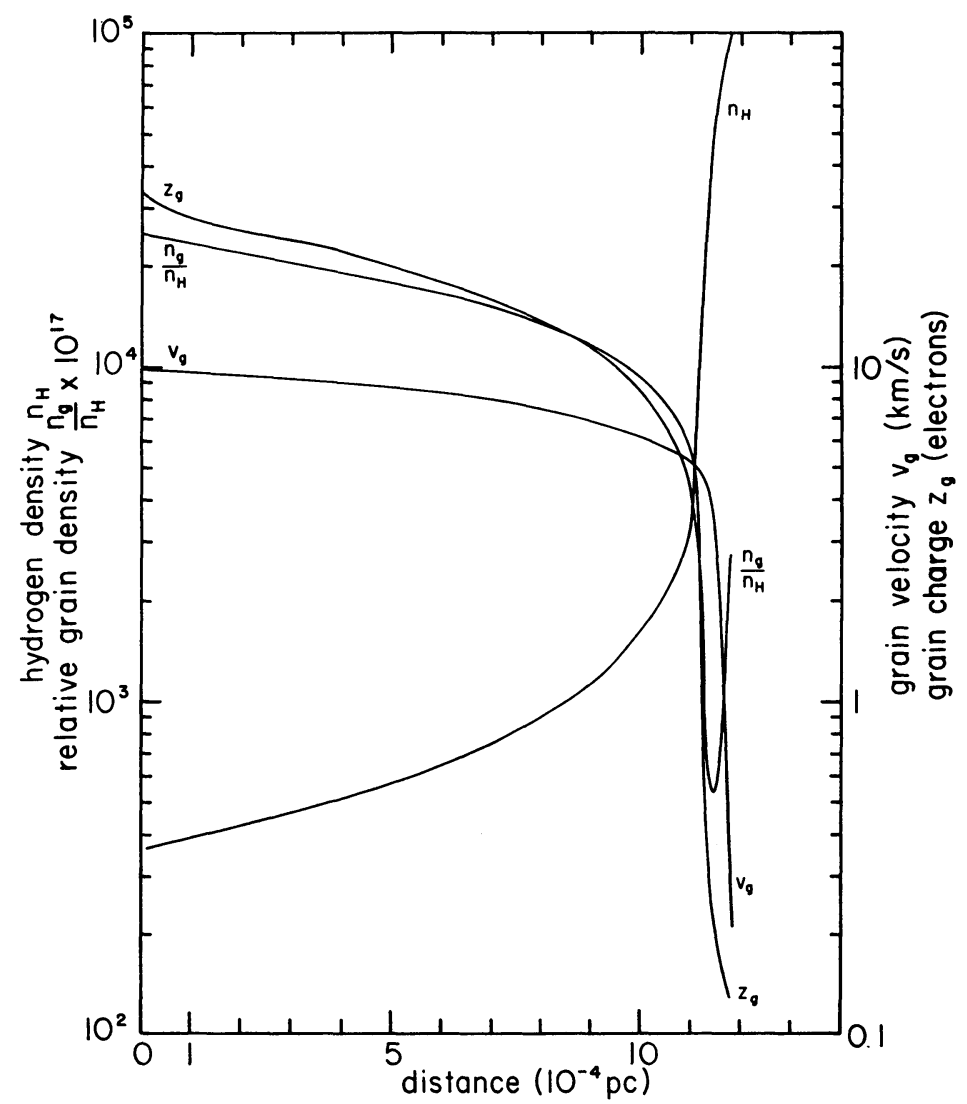

Fig. 2. The velocity of the grain with respect to the shock front, the relative grain density, the grain charge and the gas density as functions of the distance behind the shock front. The shock velocity is $10 \mathrm{~km} \mathrm{~s}^{-1}$ and $\mathrm{H}_{2} / \mathrm{H}=2 \times 10^{-2}$. 
Since the mean free path for grain-grain collisions in this region is about $1 \mathrm{pc}$ and only about $10 \%$ of the mass of a typical cloud is in the interpenetration region of two clouds, we conclude that the probability of mantle destruction due to grain-grain collision is about $10^{-4}$. This is a factor of more than $10^{3}$ smaller than the destruction probability due to the sputtering process.

Since the growth of dirty ice mantles will cause the interstellar gas to lose cooling elements, clouds may tend to heat up and make a sort of phase transition into the hot and rarified state of the intercloud gas. If we use the thermal sputtering yields for $\mathrm{He}$ and $\mathrm{H}$ sputtering of $\mathrm{H}_{2} \mathrm{O}$ shown by the solid lines in the Figure 3 (the dashed lines are for $\mathrm{CH}_{4}$ ), we find a yield of about 0.1 for an intercloud temperature of $7500 \mathrm{~K}$. With a gas density of $0.2 \mathrm{~cm}^{-3}$ a grain may be sputtered from $0.15 \mu$ to $0.05 \mu$ in about $3 \times 10^{7} \mathrm{yr}$.

It thus appears that sputtering processes are fast enough to effectively clean the interstellar ice grains if they are exposed to the conditions in the intercloud medium. Intercloud grains should thus be somewhat smaller than cloud grains. From the ob-

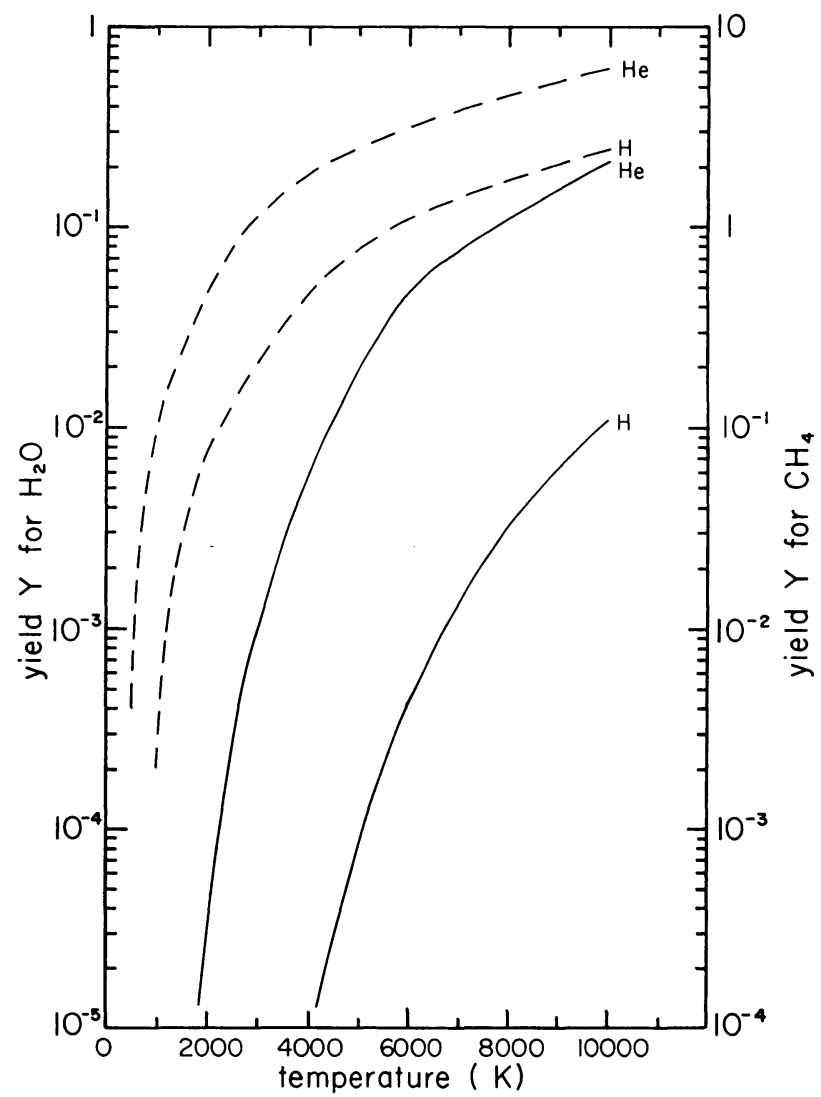

Fig. 3. Thermal sputtering yield as a function of temperature. The solid curves are for sputtering of $\mathrm{H}_{2} \mathrm{O}$, and the dashed curves for sputtering of $\mathrm{CH}_{4}$. The sputtering agents are $\mathrm{H}$ and $\mathrm{He}$ as indicated. 
servations by FitzGerald (1968), assuming a ratio of total to selective absorption of 3 , we find that the ratio of visual extinction to column density of hydrogen is at least a factor of two smaller for regions outside the clouds, indicating an effect of the kind we have proposed here.

\section{References}

FitzGerald, M. P.: 1968, Astron. J. 73, 893.

Knacke, R. F., Cudaback, D. D., and Gaustad, J. E.: 1969, Astrophys. J. 158, 151. 